\title{
Time Compression in Virtual Reality
}

\author{
Grayson Mullen $^{*, * *}$ and Nicolas Davidenko ${ }^{* * *}$ \\ Department of Psychology, University of California, Santa Cruz, CA 95060, USA
}

Received 12 August 2020; accepted 30 March 2021

\begin{abstract}
Virtual-reality (VR) users and developers have informally reported that time seems to pass more quickly while playing games in VR. We refer to this phenomenon as time compression: a longer real duration is compressed into a shorter perceived experience. To investigate this effect, we created two versions of a labyrinth-like game. The versions are identical in their content and mode of control but differ in their display type: one was designed to be played in VR, and the other on a conventional monitor (CM). Participants were asked to estimate time prospectively using an interval production method. Participants played each version of the game for a perceived five-minute interval, and the actual durations of the intervals they produced were compared between display conditions. We found that in the first block, participants in the VR condition played for an average of 72.6 more seconds than participants in the CM condition before feeling that five minutes had passed. This amounts to perceived five-minute intervals in VR containing $28.5 \%$ more actual time than perceived five-minute intervals in CM. However, the effect appeared to be reversed in the second block when participants switched display conditions, suggesting large novelty and anchoring effects, and demonstrating the importance of using between-subjects designs in interval production experiments. Overall, our results suggest that VR displays do produce a significant time compression effect. We discuss a VR-induced reduction in bodily awareness as a potential explanation for how this effect is mediated and outline some implications and suggestions for follow-up experiments.
\end{abstract}

\section{Keywords}

Virtual reality, bodily awareness, interoception, time compression, prospective time estimation, presence, immersion

\footnotetext{
* To whom correspondence should be addressed. E-mail: gmullen@ucsc.edu

** ORCID: oooo-ooo2-3324-6491

*** ORCID: oooo-ooo2-6935-5542
} 


\section{Introduction}

Virtual-reality (VR) head-mounted displays (HMDs) take up the user's entire field of view, replacing all of their real-world visual cues with a contrived virtual world. This imposes unique conditions on human vision and on all other brain functions that make use of visual information. The consequences have mostly been studied in terms of presence, or the feeling of being inside the virtual scene presented on the HMD rather than in the real world (see Heeter, 1992 for a more encompassing and widely used definition of presence). Because the virtual scene can be designed to look like anything, VR can produce unique psychological effects by placing users in situations that rarely (or never) occur naturally. For example, it can present visual stimuli that conflict with the users' vestibular cues, causing cybersickness (Davis et al., 2014). VR experiences have also been intentionally used to reduce pain in burn patients (Hoffman et al., 2011), to elicit anxiety or relaxation (Riva et al., 2007), and even to affect self-esteem and paranoia by manipulating the height of the user's perspective relative to the virtual scene (Freeman et al., 2014).

One unintentional effect, which has been anecdotally reported by VR users and developers, is a time compression phenomenon wherein a larger real duration is compressed into a shorter perceived experience. At a 2016 gaming conference, Hilmar Veigar (of CCG Games) said, "You think you've played for 15 minutes and then you go out and it's like, 'Wait, I spent an hour in there?' There's a concept of, I don't know, VR time” (Miller, 2016). Palmer Luckey (founder of Oculus) suggested that the effect could be a result of not having access to real-world environmental cues, like the position of the sun. Distorted time perception has been observed as an effect of conventional gaming (Nuyens et al., 2020), but the influence of VR on time perception has been studied relatively less.

One notable study (Schneider et al., 2011) successfully used VR experiences to shorten perceived durations during chemotherapy and found individual differences in time compression effects related to diagnosis, gender and anxiety. It is not clear, though, whether a non-VR version of the same experience would have resulted in a similar distortion of time perception. Only a few studies have directly compared time estimation processes between a VR experience and a non-VR counterpart, and none so far have found significant differences.

Bansal et al. (2019) examined the influence of a novel modification of a VR game (which coupled the flow of time to the speed of players' body movements) on participants' performance on subsequent time estimation tasks. Compared to control groups, participants who played the modified game made shorter estimates of brief ( $6 \mathrm{~s}$ and shorter) intervals, but only on estimation tasks that involved continuous movement. No significant difference in time perception was found between participants who played an unmodified (normal-time) version of the VR game and those who played a non-VR game. These results indicate that VR alone may not recalibrate temporal perception, but that a specifically tailored VR 
experience may induce such an effect. Because all the time estimation tasks were performed outside of VR, these results do not provide an answer to the question of whether time perception is distorted during VR use.

Schatzschneider et al. (2016) investigated how time estimation was affected by display type (VR/non-VR) and cognitive load. The researchers found no significant difference in time estimation between the display conditions, but the study used a within-subjects design and all participants experienced the non-VR condition first and the VR condition second. Completing the non-VR condition first may have anchored participants' time estimates in the subsequent VR condition. Thus, it is possible that the lack of counterbalancing in Schatzschneider et al. (2016) may have obscured an effect of display type. Another study (van der Ham et al., 2019) also found no difference in time estimates between VR and non-VR displays, but used a retrospective time estimation paradigm.

According to Block and Zakay (1997), retrospective and prospective time estimates depend on different processes. Retrospective estimates are made when participants are unaware that they will be asked to estimate a duration until after the interval has ended. These estimates are based only on information that is stored in memory. Factors that have been found to affect retrospective time estimates are mostly related to stimulus complexity and contextual changes (more complex information and more changes are associated with longer retrospective estimates). Because they rely on memory, retrospective time estimates are affected by cognitive load only indirectly, when information relevant to cognitive load is stored in memory.

In contrast, prospective estimates are made by participants who are aware during the interval that they will be asked to estimate its duration. The most prominent model to illustrate the processes underlying prospective time estimation is Zakay and Block's (1995) attentional-gate model of prospective time estimation (but see also Grondin, 2010; Ivry \& Schlerf, 2008; and Wittmann, 2009 for reviews of alternate models of time perception). The first component of this abstract model is a pacemaker (which can be thought of as an internal metronome) that generates pulses at a rate that scales with the estimator's arousal. Before the pulses can be counted, they are modulated by an attentional gate, which is open to a variable degree depending on the amount of attentional resources allocated to tracking time. When attentional resources are consumed by a demanding task, the gate becomes narrower (i.e., fewer resources are available to attend to time), and fewer pulses are able to pass.

The pulses that pass the attentional gate are counted by an accumulator, and the resulting sum is used as the basis for an estimate of the interval's duration. The larger the count, the more time the estimator reports has passed. This means that time seems to pass more quickly (i.e., it becomes compressed) when attentional demands are high, and it seems to pass more slowly (i.e., it dilates) when attentional demands are low. The attentional-gate model is supported by the 
preponderance of attention-related manipulations that have been found to significantly affect prospective estimates, but not retrospective estimates (Block \& Zakay, 1997). Thus, whereas prospective estimates are affected by cognitive load, retrospective estimates are more affected by contextual changes and other memory-related factors.

The current study is the first to investigate the effect of VR HMDs on time perception using a prospective time estimation paradigm and counterbalanced display conditions. We chose a prospective time estimation paradigm in order to measure the experience of VR rather than the memory of it (Block \& Zakay, 1997), and also to obtain results that are relevant to intentional time management while playing VR games. We also used an interval production method of time estimation (Zakay, 1993), in which the research assistant specifies a duration (five minutes, in our case) and the participant starts and ends an interval that they feel matches that duration. This method is less susceptible to rounding biases than methods that ask the participant to report the number of seconds or minutes an interval lasted. In our study, every participant attempts to produce a five-minute interval, and we use the actual durations of the intervals they produce as our main dependent variable.

\subsection{Hypotheses}

First, we predict that intervals produced while playing a VR game will be longer than those produced while playing an equivalent game displayed on a conventional monitor (CM). This hypothesis is based on the anecdotal reports of a time compression effect in VR, and is motivated by past studies which have probed the relationship between time perception and VR but failed to find evidence of this effect. Based on Block and Zakay's (1997) comparison of time estimation methods, we expect an interval production method to yield evidence of a compression effect in VR that has not been directly revealed by other methods.

Second, we predict that VR interval durations will be more variable across participants than CM interval durations. Higher variability is naturally expected if VR interval durations are longer, assuming that errors are proportional to the size of the estimate. Additionally, we predict that variability may be further increased by uncertainty in time perception among participants in VR. If VR interferes with normal time perception, participants may be less confident in their ability to track the passage of time, and produce a wider range of interval durations.

\section{Methods}

\subsection{Participants}

Forty-one undergraduate students participated for course credit. Two of them produced extreme outlier responses (their intervals in the VR condition were 
more than three standard deviations above the mean), so our final analysis includes data from 39 participants (24 female and 15 male, ages $18-26, M=19.5$, $\mathrm{SD}=1.7)$. The UC Santa Cruz IRB approved the study and participants provided informed consent.

\subsection{Materials}

In both conditions, participants played a 3D labyrinth-like game designed in Unity. Each level consisted of a floating maze inside an otherwise empty room with textured walls and floors (see Fig. 1). The lighting and object textures did not change between levels, conditions, or maze sets, and there was no representation of the user's body. The maze was positioned in front of and below the virtual camera to allow participants to see into the maze from above. Each maze contained a ball and a glowing yellow cube representing a goal, as well as walls and holes in the floor. Participants were directed to guide the ball to the goal by tilting the maze. Each version of the game included one of two maze sets (designed to be equally complex and difficult) so that participants did not repeat any levels between the two conditions. Each version included one practice level followed by up to 13 timed levels, which became increasingly difficult to complete as the mazes became larger and more complex (to simulate the general sense of progression in video games). Letting the ball fall through a hole in the maze would restart the current level, while getting the ball to reach the goal would start the next level. Above the maze in the timed levels, white text reading, "When you think five minutes have passed, press the right bumper and trigger at the same time" continuously faded in and out on an 8-s cycle to remind participants of the interval production task.

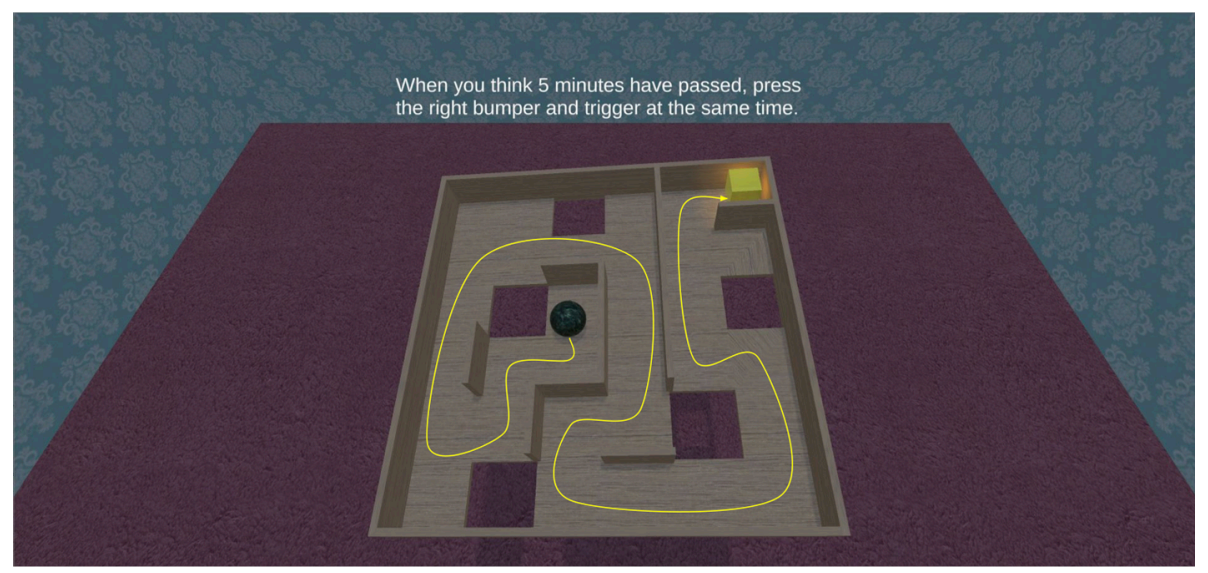

Figure 1. The sixth level of maze set $A$ as viewed by participants in both the virtual reality (VR) and conventional monitor (CM) condition. The superimposed yellow line (not shown to participants) indicates a path to the goal. 
We decided it was important to include this reminder because when using an interval production method, the interval does not end until the participant chooses to end it. If a participant forgets that they were asked to keep track of time, they could produce exceedingly long intervals that are not accurately descriptive of their perception of time. Although the periodic fading of the reminder may have served as a temporal cue to make time perception more accurate, we do not expect it to have confounded our results because it was presented the same way in the VR and CM conditions of the game.

Participants used an Xbox 360 controller (Microsoft Corporation; Redmond, WA, USA) to manipulate the maze. They could tilt it in eight directions by moving the left joystick and could return it to its original position by holding any of the colored buttons (A, B, X, or Y). The right trigger and bumper (buttons at the back of the controller) were pressed simultaneously to end the practice level, and later to indicate the end the perceived 5-min interval.

In the VR condition, participants wore an Oculus Rift CV1 HMD (Oculus VR; Menlo Park, CA, USA) with head-tracking enabled to show a stable 3D environment. In the CM condition, participants viewed the game on a 20-inch Dell monitor with a $1920 \times 1080$ pixel resolution and a $60 \mathrm{~Hz}$ refresh rate. Participants in the CM condition were seated approximately $45 \mathrm{~cm}$ away from the monitor. At this distance, the maze subtended approximately 22 degrees by 22 degrees of visual angle. Participants in the VR condition saw the maze from a virtual camera that was positioned similarly with respect to the maze, but the maze subtended a slightly larger visual angle (approximately 30 degrees by 30 degrees). However, participants were allowed to move freely during the game in both conditions, so the visual angle of the maze varied considerably across participants and across maze levels. Other than these differences between displays, the game was played on the same computer hardware between conditions.

After completing both conditions, participants filled out a questionnaire that asked about the difficulty of tracking time and of playing the game, their confidence in their ability to estimate time, previous experience with VR and video games, and included 19 Likert-scale items about immersion (e.g., "I felt detached from the outside world"). The purpose of this immersion scale was to measure whether participants felt significantly more immersed in the VR condition compared to the CM condition, and to show if immersion played a mediating role in any time compression effect we might find.

\subsection{Procedure}

We used a counterbalanced within-subjects design because we expected time perception accuracy to be highly variable between people. There were two display conditions (virtual reality [VR] and conventional monitor [CM]) as well as two sets of mazes (A and B). Each participant played the game once in VR and once on the $\mathrm{CM}$, one of which used maze set $\mathrm{A}$ and the other used set B. Display condition 
and maze set were both counterbalanced to minimize order and maze difficulty effects.

Participants were asked to keep their phones and watches out of sight for the duration of the experiment, and to sit in front of a computer at a desk in our lab room. No clocks were visible to the participants, and research assistants in adjacent rooms refrained from using time-related language. Figure 2 illustrates the equipment used in each condition. A research assistant read instructions on how to play the game, and the practice level was started while the controls were described. Participants were told they could play the practice level for as long as they wanted to get comfortable with the game, and that it was not timed. Once they were ready to stop practicing, they could start the timed levels, which they were instructed to end once they felt they had been playing for five minutes. The research assistant left the room and shut the door after the instructions to minimize distractions and aural cues from outside the room.

We chose not to vary the duration of the intervals that participants were instructed to produce because of our limited sample size. We set the target duration at five minutes because it is a familiar and memorable unit of time, and we expected it would be long enough to discourage deliberate counting of seconds, but short enough to minimize fatigue effects (especially in the second sessions).

When the participant ended the timed levels, the elapsed time in seconds since the end of the practice level was automatically recorded in a text file, along with their practice time and the level that the participant had reached. No feedback about how much time had actually passed was given to the participant. Then, the research assistant briefly reminded the participant of the instructions and started the second game, which used the display condition and maze set that were not used in the first game.
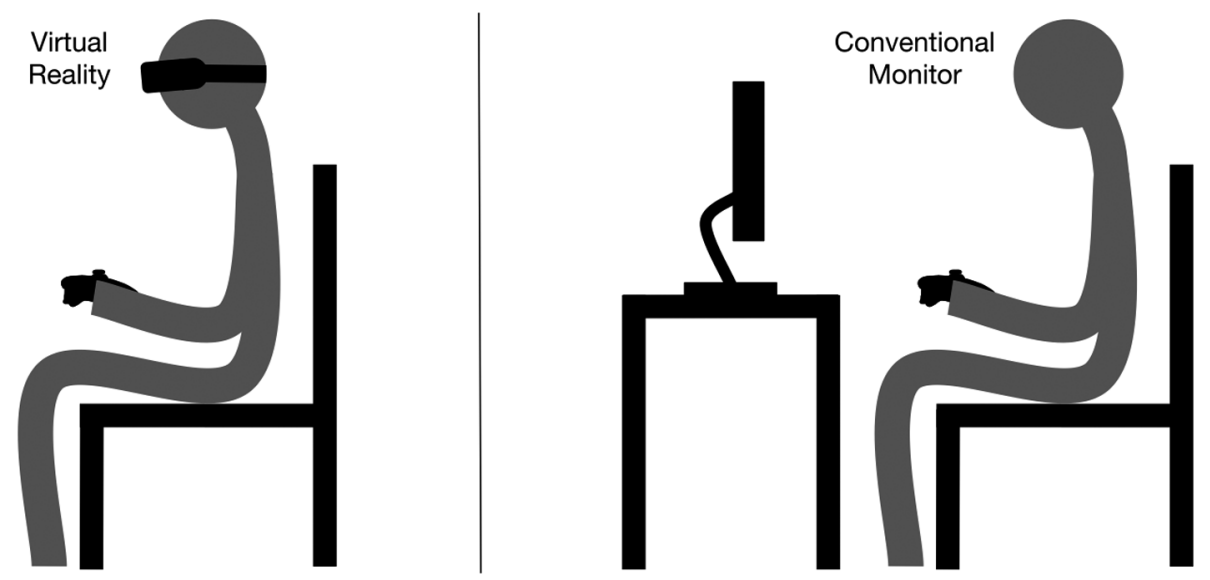

Figure 2. Illustrations of the virtual reality and conventional monitor display conditions. 
After both versions of the game were completed, the participant was brought to a new room to complete a post-task survey (see Materials above).

\section{Results}

We conducted a two-way mixed-effects ANOVA with factors of starting display type (VR or CM) and block number (first or second). The results, shown in Fig.3, revealed a main effect of block number $\left(F_{1,37}=9.94, p=0.003, \eta p^{2}=0.212\right)$, indicating that the mean duration of intervals produced in the second block (341.9 s) was significantly longer than that of intervals produced in the first block (290.1 s). Importantly, there was a main effect of starting display type $\left(F_{1,37}=6.45\right.$, $p=0.015, \eta p^{2}=0.148$ ). Participants who played the VR game first (and the CM game second) produced longer intervals than participants who played the CM game first (and the VR game second). This means that the effect of display type on interval duration depends on order: in the first block, participants in the VR condition produced longer durations (327.4s on average) than participants in the CM condition (254.8 s), whereas in the second block, VR durations (299.9 s) were shorter than CM durations (386.2 s). Furthermore, we found a strong correlation between participants' first and second interval durations $(r=0.62, p<0.001$,

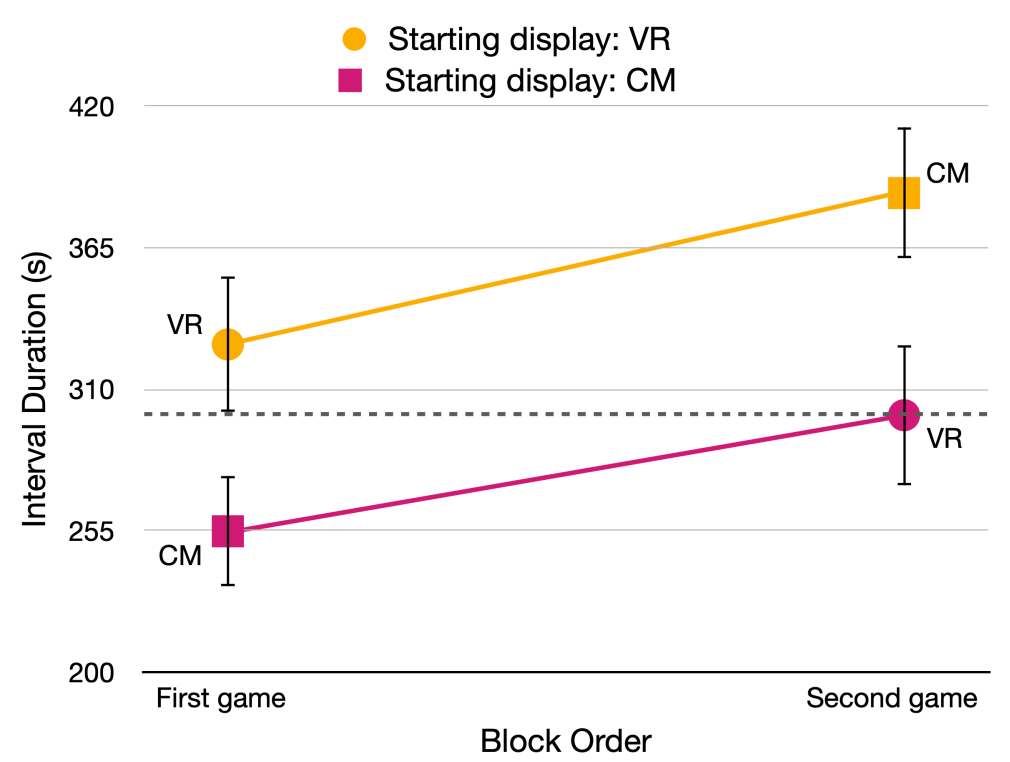

Figure 3. A line graph showing mean produced interval durations (with standard error bars) organized by condition and starting display. Participants who started with virtual reality (VR) produced longer intervals in both the first and second blocks, and second block intervals were longer than first-block intervals. The dotted horizontal reference line shows the position of an accurate interval. $\mathrm{CM}$, conventional monitor. 
$n=39$ ), suggesting individuals' second intervals were heavily anchored to their first ones. Because of this order effect, we limit our remaining analyses to firstblock responses.

As shown in Fig. 4, first-block participants in the VR condition let significantly more time pass than first-block participants in the $\mathrm{CM}$ condition before indicating that five minutes had passed $\left(t_{37}=2.165, p=0.037, d=0.693\right)$. VR intervals were $327.4 \mathrm{~s}$ long $(\mathrm{SD}=114.0)$ on average, and CM intervals were $254.8 \mathrm{~s}(\mathrm{SD}=95.1)$ on average. This means that in the VR condition, 72.6 more seconds (95\% CI, [4.6, 140.6]) passed on average before participants felt that five minutes had elapsed. This finding supports our first hypothesis, that participants experience time compression in VR compared to playing an identical game on a CM.

To rule out an account based on differences in task difficulty, we compared how quickly participants in the two conditions completed the levels of the maze game. Figure 5 shows that the relationship between interval duration and level reached is described by a similar linear relationship in the two conditions. To determine whether these slopes were significantly different, we ran 10,000 bootstrap samples from each condition to compare the resulting best-fit lines and found that the $95 \%$ confidence interval for the difference between best-fit slopes in the VR and the CM condition [ $-0.0021,0.0072]$ contained zero. Therefore participants across the VR and CM conditions completed levels at similar rates, suggesting that the time compression effect cannot be attributed to participants spending more time on each level in VR compared to CM and using the number of levels completed as a proxy to decide when five minutes had elapsed. Furthermore we

420

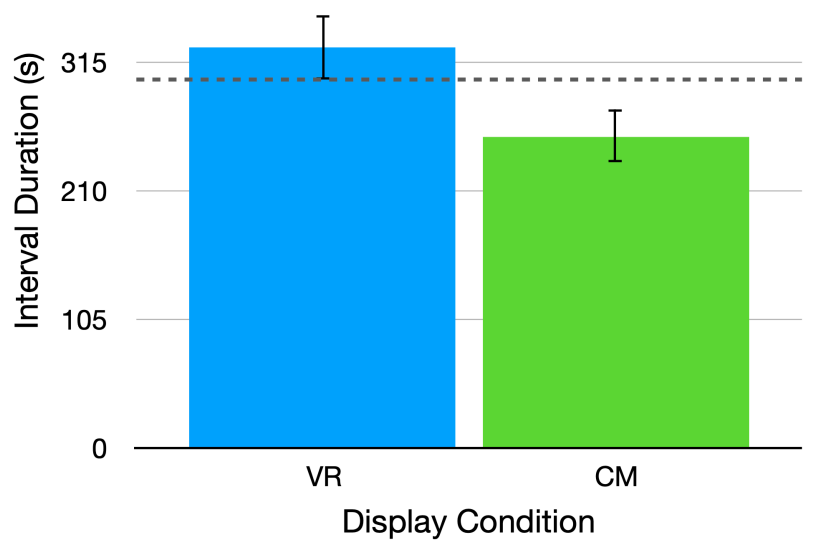

Figure 4. A bar graph showing mean interval durations produced in the virtual reality (VR) and conventional monitor (CM) conditions (first block only) with standard error bars. Participants in the VR condition produced significantly longer intervals. The horizontal reference line shows the position of a true 5-min interval. 


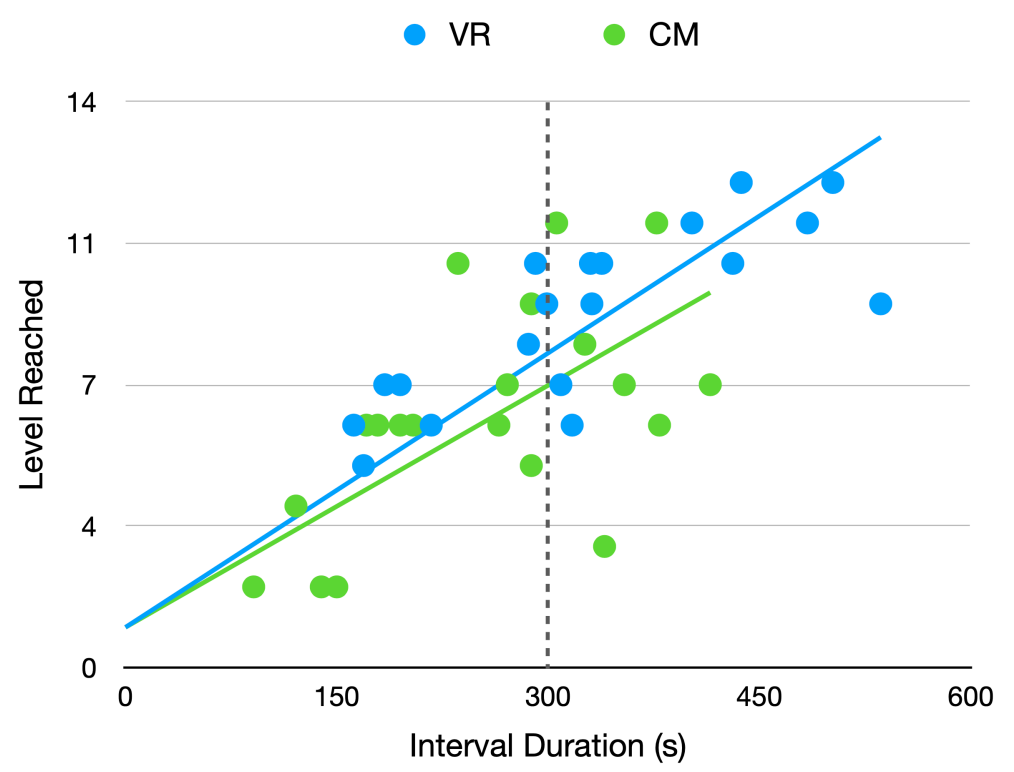

Figure 5. A scatterplot of each participant's interval duration and the number of levels they reached in the first experiment block. The best-fit lines have a fixed $y$-intercept at 1 (a 0 -s time interval would correspond to level 1), and the vertical reference line shows the position of an accurate interval.

found no significant difference in practice time between conditions $\left(t_{37}=-0.147\right.$ $p>0.5, d=0.047)$ suggesting it was not more difficult to learn the game in VR than in CM.

We did not find support for the hypothesis that produced interval durations would be more variable in the VR condition. Although intervals produced in the VR condition $(\mathrm{SD}=114 \mathrm{~s})$ were slightly more variable than intervals produced in the $\mathrm{CM}$ condition ( $\mathrm{SD}=95 \mathrm{~s}$ ), Levene's test showed that there was no significant difference in interval variance between conditions $\left(F_{1,37}=0.195, p>0.5\right)$.

The survey responses did not reveal a significant relationship between interval durations and previous experience with video games or with VR, nor was there a significant difference between conditions in rated difficulty (either of the game or of keeping track of time). This result conflicts with our second prediction that time estimation in VR would be more difficult, and that produced intervals would therefore be more variable in VR compared to CM. However, because the survey was administered after participants had completed both tasks, it is possible that participants' responses pertaining to one condition were confounded by their experience with the other. In fact, we found no significant differences in ratings of immersion between the VR and CM conditions. Only one of the 19 Likert scales about immersion ("I did not feel like I was in the real world but the game world") appeared to be higher in VR compared to $\mathrm{CM}\left(t_{36}=2.215, p=0.033, d=0.717\right)$, but this difference did not reach significance at the Bonferroni-corrected alpha of 
0.0026 (see Supplementary Table S1 for the complete immersion scale results). The surprising lack of an immersion difference between conditions suggests that administering the survey after both conditions were completed may have diminished our ability to detect an effect.

\section{Discussion}

These results constitute the first evidence that $\mathrm{VR}$ as a medium produces a unique time compression effect. At least one previous experiment (Schneider et al., 2011) successfully used VR to produce a similar effect, but the present study is the first to observe time compression as a significant difference between VR and non-VR experiences with otherwise identical content. Importantly, our results suggest that there is something inherent about the VR interface (as opposed to a characteristic of its content) that produces a time compression effect.

Most of the previously observed effects on prospective time estimation are related to attention, but the significance of our main finding does not appear to be attributable to a difference in attentional demands. The tasks in both conditions were of identical complexity and difficulty; the two sets of maze levels were counterbalanced across conditions, and participants in both conditions spent about the same amount of time on each level.

The VR condition did present a simpler scene to the participant than the CM condition (it had a narrower field of view, and the physical lab environment was not visible), but this is unlikely to explain our effect either. Visual-stimulus complexity has been found to only affect retrospective estimates (Block \& Zakay, 1997). If we were to repeat this experiment using retrospective estimates, we would expect to find shorter perceived intervals in the VR condition, because the VR scene presents a smaller amount of information that could be later recalled from memory. This would also be a kind of time compression effect, but assuming that the participants' attention remains on the screen during the interval, we would expect a much weaker effect than the one we found. Based on Block and Zakay's (1997) meta-analysis, though, stimulus complexity should have no significant effect on prospective estimation tasks like the one we used.

Arousal can also influence prospective time estimation in general, but it is highly unlikely to explain our main finding because of the direction of its effect. Images displayed in VR have been found to elicit higher arousal than the same images displayed on conventional monitors (Estupiñán et al., 2014), but higher arousal is associated with time dilation, according to the attentional-gate model (Zakay \& Block, 1995). In the context of our study, this would predict that participants in the VR condition would produce shorter intervals than participants in the CM condition. Because produced intervals in the VR condition were in fact longer, we conclude that arousal did not play a role in the main effect we observed, either. 
One difference between our two conditions that does seem likely to be responsible for the effect is that participants could not see their own body, or any representation of it, in the VR condition. In pacemaker-accumulator models of time perception, pulse generation is treated as an abstract module of the time estimation process, but it is thought to be a function of bodily rhythms like heart rate, breathing, or neural oscillations (Pollatos et al., 2014; Wittmann, 2009). The model's inclusion of arousal as an influence on the pacemaker is based on this assumption, and there is accumulating evidence that time estimation accuracy is dependent on awareness of bodily rhythms. It has been found that time estimation accuracy is significantly correlated both with ability to estimate one's own heart rate (Meissner \& Wittmann, 2011), and with heart rate variability itself (Cellini et al, 2015). A more recent study found that people with high interoceptive accuracy are less susceptible to emotion-induced distortions of time perception (Özoğlu \& Thomaschke, 2020).

Bodily awareness was measured as a participant variable in those studies, but it can also be manipulated. An experiment which used a VR and non-VR version of the same interactive environment found that bodily awareness was reduced in VR (Murray \& Gordon, 2001). Specifically, the participants in the VR condition gave significantly lower ratings on scales of cardiovascular, skin, and muscle awareness. This is presumably related to the absence of any visible representation of the users' body in the VR scene.

The combination of these two findings, (1) that prospective time estimation accuracy is related to awareness of bodily rhythms and (2) that being in VR reduces bodily awareness, suggests a likely explanation for the effect observed in the current study: participants in the VR condition were less aware of the passage of time because they were less aware of the bodily rhythms that form the basis of prospective time perception.

This is notable because the most prominent models of prospective time estimation do not account for interoceptive awareness as an independent influence on perceived interval durations. For example, pacemaker-accumulator models like Zakay and Block's (1995) attentional gate include arousal, attention, and reference memory - but not interoceptive awareness - as influences on prospective time estimation. Because we suspect that a difference in interoceptive awareness (and not in attention, arousal, or memory) best explains the VR-induced time compression effect, models like these might be modified to account for interoceptive awareness as an independent influence on prospective time estimation. Dedicated timing models (Ivry \& Schlerf, 2008) such as the attentional-gate model involve a pacemaker module that produces pulses that depend on bodily rhythms such as heart rate, breathing, or neural oscillations. We propose that such models might be amended to include interoceptive awareness as a factor that mediates the reception of these pulses. Impairing interoceptive awareness would lead to underestimations of time by reducing the number of pulses that ultimately reach 
the accumulator. Although prominent models so far have not treated interoceptive awareness as its own factor, our results suggest that it may affect time estimation independently from attentional demands, arousal, and reference memory.

The durations of participants' second intervals were heavily anchored to first interval durations. It could be that the time production task in the first block severely revised each participants' reference for what a five-minute interval feels like, and caused them to use that new reference to produce the second interval. Second intervals were also longer. This effect was exhibited by participants who played the VR version first and then switched to CM, as well as those who started with CM and switched to VR. The greater durations of second block intervals could be due to a novelty effect which may have dilated time perception more during the first block compared to the second block. Alternatively, participants may have expected to complete more levels in a 5-min period during the second block after having gained experience with the task. If participants expected to complete more levels in the second block, and used the level reached in the first block as a proxy to indicate the passing of five minutes, they may have purposely played additional levels in the second block. In fact, participants did on average play one additional level in the second block, but the rate of completing levels was no faster compared to the first block.

It is well established that order effects in general can confound results when counterbalancing is not used, but in our case the order effect was so overwhelming that the time compression effect becomes completely obscured if we analyze our data without regard for condition order. This suggests that counterbalancing may not be sufficient for experiments which use interval production tasks, and that future studies should use between-subjects designs when possible.

A follow-up experiment could further investigate the role of interoception in VR-induced time compression by having participants complete a bodily awareness scale after they complete the maze game. Using a between-subjects design in such an experiment would allow the questionnaire to be administered immediately after a single playthrough of the maze game, making it more valid than ours (which was administered after participants had completed both conditions).

Including an additional VR condition with a virtual body representation could also help clarify the role of body visibility in time perception (and more broadly, in bodily awareness). It is unclear now if hiding one's body from view is enough to reduce bodily awareness, or if the effect depends on the VR-induced feeling of presence that makes the user feel as though they are in a place that is remote from their body. If adding a virtual body were found to both increase bodily awareness and mitigate the time compression effect, that would support the idea that reduced body visibility is responsible for the main effect we observed. If that manipulation were found to have no impact on bodily awareness or the time compression effect, it would suggest that the effect depends not on body visibility but on some higher-level feeling of virtual presence. 
Another limitation of the present experiment is that we did not vary the duration of the interval that participants were asked to produce. Bisson and Grondin (2013) and Tobin et al. (2010) found that during gaming and internet-surfing tasks, significant time compression effects were only evident during longer sessions (around $30 \mathrm{~min}$ or longer). The authors of those studies note that this difference may be due to the time estimation methods they used: participants were asked to verbally estimate durations, and might have rounded their answers to multiples of five minutes. This rounding bias would have a much stronger influence on the results of their shorter-interval trials (12 min) than on their longerinterval trials $(35,42$, or $58 \mathrm{~min})$. Our finding of a time compression effect on a five-minute scale suggests that the interval production method we used likely protected our results from such a rounding bias. It is unclear whether or how the VR-induced effect we found might depend on the target duration of the produced interval. Future studies investigating this effect could explore this influence by instructing participants in different conditions to produce intervals shorter and longer than five minutes.

If transient reminders like the one we used are employed during prospective time estimation tasks, we recommend that the durations of the interval be pseudo-randomized. Our reliably periodic reminder may have helped our participants produce more accurate intervals in both conditions. Making the cue unreliable might reveal a larger effect, which could be crucial in experiments that test time perception in more delicate contexts.

\subsection{Implications for VR Experience Design}

An average of $28.5 \%$ more real time passed for participants who played the VR game than for those in the control group - with no difference in perceived duration. If this effect proves to generalize to other contexts at similar magnitudes, it will have significant implications. Keeping track of time accurately is desirable in most situations, and impairing that ability could be harmful.

Time compression might cause VR users to unintentionally spend excessive amounts of time in games, especially as HMDs become more comfortable to wear for long sessions. Even non-immersive games entail some risk of addiction, which has been associated with depression and insomnia (Kuss \& Griffiths, 2012). VR games may pose a greater risk of interfering with their players' sleep schedules, mood, and health by reducing their ability to notice the passage of time. Developers should take care not to create virtual 'casinos'; a clock should always be easily accessible, and perhaps even appear automatically at regular intervals.

On the other hand, time compression effects can be desirable in situations that are unpleasant but necessary, and there are potential applications that could take advantage of the effect in a beneficial way. VR might be used, for example, to reduce the perceived duration of long-distance travel. More importantly, the 
value of using VR to make chemotherapy more bearable (Schneider et al., 2011) is supported by the current study. Especially considering that VR has been used successfully as an analgesic (Hoffman et al., 2011), VR experiences could be similarly applied to reduce the negative psychological impact of other painful medical treatments. Our interpretation of the results suggests that other equipment or treatments which reduce bodily awareness, such as sensory deprivation tanks, may also be useful for producing time compression effects.

\section{Supplementary Material}

Supplementary material is available online at:

https://doi.org/10.6084/m9.figshare.14339438

\section{References}

Bansal, A., Weech, S., \& Barnett-Cowan, M. (2019). Movement-contingent time flow in virtual reality causes temporal recalibration. Sci. Rep., 9, 4378. doi: 10.1038/s41598-019-40870-6.

Bisson, N., \& Grondin, S. (2013). Time estimates of internet surfing and video gaming. Timing Time Percept., 1, 39-64. doi: 10.1163/22134468-00002002.

Block, R.A., \& Zakay, D. (1997). Prospective and retrospective duration judgments: A meta-analytic review. Psychon. Bull. Rev., 4, 184-197. doi: 10.3758/BF03209393.

Cellini, N., Mioni, G., Levorato, I., Grondin, S., Stablum, F., \& Sarlo, M. (2015). Heart rate variability helps tracking time more accurately. Brain Cogn., 101, 57-63. doi: 10.1016/j.bandc.2015.10.003.

Davis, S., Nesbitt, K., \& Nalivaiko, E. (2014). A systematic review of cybersickness. Proc 2014 Conf. Interact. Entertain., 1-9. doi: 10.1145/2677758.2677780.

Estupiñán, S., Rebelo, F., Noriega, P., Ferreira, C., \& Duarte, E. (2014). Can virtual reality increase emotional responses (arousal and valence)? A pilot study. In A. Marcus (Ed.), Design, User Experience, and Usability. User Experience Design for Diverse Interaction Platforms and Environments. DUXU 2014. Lecture Notes in Computer Science, Vol. 8518, pp. 541-549. Springer, Cham, Switzerland. doi: 10.1007/978-3-319-07626-3_51.

Freeman, D., Evans, N., Lister, R., Antley, A., Dunn, G., \& Slater, M. (2014). Height, social comparison, and paranoia: An immersive virtual reality experimental study. Psychiat. Res., 218, 348-352. doi: 10.1016/j.psychres.2013.12.014.

Grondin, S. (2010). Timing and time perception: a review of recent behavioral and neuroscience findings and theoretical directions. Atten. Percept. Psychophys., 72, 561-582. doi: 10.3758/ APP.72.3.561.

Heeter, C. (1992). Being there: the subjective experience of presence. Presence (Camb.), 1, 262-271. doi: 10.1162/pres.1992.1.2.262.

Hoffman, H. G., Chambers, G. T., Meyer, III, W. J., Arceneaux, L. L., Russell, W. J., Seibel, E. J., Richards, T. L., Sharar, S. R., \& Patterson, D. R. (2011). Virtual reality as an adjunctive non-pharmacologic analgesic for acute burn pain during medical procedures. Ann. Behav. Med., 41, 183-191. doi: 10.1007/s12160-010-9248-7.

Ivry, R. B., \& Schlerf, J. E. (2008). Dedicated and intrinsic models of time perception. Trends Cogn. Sci., 12, 273-280. doi: 10.1016/j.tics.2008.04.002. 
Kuss, D. J., \& Griffiths, M. D. (2012). Internet gaming addiction: a systematic review of empirical research. Int. J. Ment. Health Addict., 10, 278-296. doi: 10.1007/s11469-011-9318-5.

Meissner, K., \& Wittmann, M. (2011). Body signals, cardiac awareness, and the perception of time. Biol. Psychol., 86, 289-297. doi: 10.1016/j.biopsycho.2011.01.001.

Miller, R. (2016) Oculus founder thinks VR may affect your ability to perceive time passing. The Verge. https://www.theverge.com/2016/3/17/11258718/palmer-luckey-oculus-time-vrvirtual-reality-gdc-2016.

Murray, C. D., \& Gordon, M. S. (2001). Changes in bodily awareness induced by immersive virtual reality. CyberPsychol. Behav., 4, 365-371. doi: 10.1089/109493101300210268.

Nuyens, F. M., Kuss, D. J., Lopez-Fernandez, O., \& Griffiths, M.D. (2020). The potential interaction between time perception and gaming: A narrative review. Int. J. Ment.l Health Addict., 18, $1226-$ 1246. doi: 10.1007/s11469-019-00121-1.

Özoğlu, E., \& Thomaschke, R. (2020). Knowing your heart reduces emotion-induced time dilation. Timing Time Percept., 8, 299-315. doi: 10.1163/22134468-bja10016.

Pollatos, O., Yeldesbay, A., Pikovsky, A., \& Rosenblum, M. (2014). How much time has passed? Ask your heart. Front. Neurorobot., 8, 15. doi: 10.3389/fnbot.2014.00015.

Riva, G., Mantovani, F., Capideville, C. S., Preziosa, A., Morganti, F., Villani, D., Gaggioli, A., Botella, C., \& Alcañiz, M. (2007). Affective interactions using virtual reality: the link between presence and emotions. CyberPsychol. Behav., 10, 45-56, doi: 10.1089/cpb.2006.9993.

Schatzschneider, C., Bruder, G., \& Steinicke, F. (2016). Who turned the clock? Effects of manipulated zeitgebers, cognitive load and immersion on time estimation. IEEE Trans. Vis. Comput. Graph., 22(4), 1387-1395. doi: 10.1109/tvcg.2016.2518137.

Schneider, S. M., Kisby, C. K. \& Flint, E. P. (2011). Effect of virtual reality on time perception in patients receiving chemotherapy. Support. Care Cancer, 19, 555-564. doi: 10.1007/ S00520-010-0852-7.

Tobin, S., Bisson, N., \& Grondin, S. (2010). An ecological approach to prospective and retrospective timing of long durations: a study involving gamers. PloS ONE 5, e9271. doi: 10.1371/journal. pone.0009271.

van der Ham, I. J. M., Klaassen, F., van Schie, K., \& Cuperus, A. (2019). Elapsed time estimates in virtual reality and the physical world: the role of arousal and emotional valence. Comput. Hum. Behav., 94, 77-81. doi: 10.1016/j.chb.2019.01.005.

Wittmann, M. (2009). The inner experience of time. Philos. Trans. R. Soc. B Biol. Sci., 364, 1955-1967. doi: 10.1098/rstb.2009.0003.

Zakay, D. (1993). Time estimation methods -Do they influence prospective duration estimates?. Perception, 22, 91-101. doi: 10.1068/p220091.

Zakay, D., \& Block, R. A. (1995). An attentional-gate model of prospective time estimation. In M. Richelle, V. D. Keyser, G. d'Ydewalle \& A. Vandierendonck (Eds), Time and the dynamic control of behavior (pp. 167-178). Liège, Belgium: Université de Liège. 